\title{
Effects of Histidine-Rich Glycoprotein on Erythrocyte Aggregation and Hemolysis: Implications for a Role under Septic Conditions
}

\author{
Hui Zhong ${ }^{1}$, Hidenori Wake ${ }^{1}$, Keyue Liu', Yuan Gao ${ }^{1}$, Kiyoshi Teshigawara ${ }^{1}$, Masakiyo Sakaguchi ${ }^{2}$, \\ Shuji Mori ${ }^{3}$, Masahiro Nishibori ${ }^{1}$
}

${ }^{I}$ Pharmacology Department, Graduate School of Medicine Dentistry and Pharmaceutical Sciences, Okayama University, Japan, ${ }^{2}$ Department of Cell Biology, Okayama University Graduate School of Medicine, Dentistry and Pharmaceutical Sciences, 2-5-1 Shikata-cho, Kita-ku, Okayama 700-8558, Japan, ${ }^{3}$ School of Pharmacy, Shujitsu University, 1-6-1 Nishikawahara, Naka-ku, Okayama 703-8516, Japan

Background: The apoptotic process of erythrocytes is known as eryptosis, and is characterized by phosphatidylserine (PS) expression on the outer membrane. PS-positive erythrocytes are increased in sepsis, and PS is believed to facilitate aggregation of erythrocytes and activate macrophages. However, the relationship between eryptosis and abnormal coagulation in sepsis is still not fully understood. Histidine-rich glycoprotein (HRG) is a $75-\mathrm{kDa}$ single chain protein produced mainly in the liver. In the previous study, we demonstrated that HRG conferred protection against lethality in septic mice by suppressing immunothrombosis and relevant inflammation. However, it is still not clear whether HRG suppresses immunothrombosis through regulation of erythrocytes.

Methods: $\mathrm{Zn}^{2+}$ was used to induce erythrocyte aggregation and adhesion to endothelial cells. Erythrocyte PS expression after $\mathrm{Zn}^{2+}$ stimulation was determined by FACS. Sepsis was induced by Cecal Ligation Puncture (CLP) in mice. Hemoglobin levels in plasma were determined by Western blot and $\mathrm{Zn}^{2+}$ contents in tissues were determined by commercial kit. Binding between HRG and hemin was determined by calorimetry.

Results: In the present study, we subjected isolated erythrocytes to $\mathrm{Zn}^{2+}$ stimulation, which activated their aggregation and PS expression. $\mathrm{Zn}^{2+}$-induced aggregation and PS expression in erythrocytes were inhibited by physiological concentration of HRG $(1 \mu \mathrm{M})$. Erythrocyte adhesion to endothelial cells was also significantly increased after $\mathrm{Zn}^{2+}$ stimulation, and this effect was inhibited by HRG. Finally, we examined HRG treatment in septic model mice, and found that HRG decreased hemolysis, possibly due to its ability to bind heme.

Conclusions: Our study demonstrated a novel $\mathrm{Zn}^{2+}$-initiated aggregation of erythrocytes, probably contributing to intravascular thrombus formation. We also showed the regulatory role of HRG in this pathway, together with the ability of HRG to inhibit hemolysis under septic conditions. HRG supplementation might be a novel therapeutic strategy for systemic inflammatory disorders, such as sepsis. 